
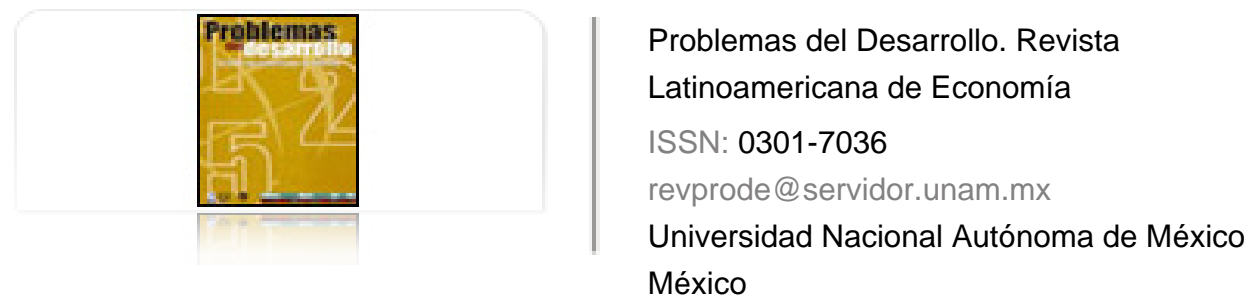

Álvarez Peralta, Ignacio

Financiarización económica y relación salarial en las economías desarrolladas Problemas del Desarrollo. Revista Latinoamericana de Economía, vol. 39, núm. 155, octubrediciembre, 2008, pp. 77-100

Universidad Nacional Autónoma de México

Distrito Federal, México

Disponible en: http://www.redalyc.org/articulo.oa?id=11820123005

- Cómo citar el artículo

- Número completo

- Más información del artículo

Página de la revista en redalyc.org

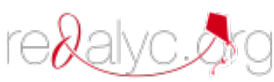

Sistema de Información Científica

Red de Revistas Científicas de América Latina, el Caribe, España y Portugal Proyecto académico sin fines de lucro, desarrollado bajo la iniciativa de acceso abierto 


\title{
FINANCIARIZACION ECONÓMICA Y RELACIÓN SALARIAL EN LAS ECONOMÍAS DESARROLLADAS
}

\author{
Ignacio Álvarez Peralta*
}

Fecha de recepción: 26 de junio de 2008. Fecha de aceptación: 30 de septiembre de 2008.

\section{Resumen}

El presente trabajo revisa el debate y la controversia actual acerca de los vínculos del proceso de financiarización económica con la relación salarial. Nuestra intención es identificar y valorar las debilidades y carencias en dicho debate, y resaltar qué aportaciones y elementos relevantes pueden tomarse para contribuir a la clarificación de dichos vínculos. Identificamos como interlocutores directos a aquellos autores y escuelas que sitúan el epicentro de su objeto de estudio en la empresa. En concreto, se revisan de forma crítica las posiciones de los autores poskeynesianos, regulacionistas, marxistas, institucionalistas así como de otros autores procedentes del campo de la economía de la empresa. Esta revisión crítica de la literatura incorpora además una propuesta interpretativa y una contrastación empírica propia para los casos de la economía estadounidense y francesa, que permiten fundamentar mejor nuestras conclusiones.

Palabras clave: financiarización, relación salarial, accionariado salarial, tasa de beneficios y acumulación.

\section{Summary}

This study reviews the debate and the present controversy over the links relating the economic financialization process with the wage relation. Our intention is to identify and evaluate the weaknesses and omissions in this debate, and to stress that the contributions and relevant elements can be taken to contribute towards the clarification of these links. We identify as direct spokesmen those authors and schools who locate the epicenter of their objective of study in the firm. Concretely, we critically review the positions of the post-Keynesians, "regulationists", Marxists, "institutionalists", and other writers in the field of the economics of the firm. This critical review of the literature also incorporates an interpretative proposal and an empirical comparison from specific cases in the U.S. and French economies, to provide a better basis for our conclusions.

Key words: financialization, wage relation, shareholders' earnings, rate of benefits and accumulation.

* Investigador del Departamento de Economía Aplicada I, Universidad Complutense de Madrid. Correo electrónico: nacho.alvarez@icei.ucm.es. 
Résumé

Dans ce travail sont examinés les termes du débat et de la controverse actuels relatifs aux liens entre le processus de financiarisation économique et la relation salariale. Notre intention est d'identifier et d'évaluer les faiblesses et carences de ce débat, et de souligner les apports et éléments importants qui peuvent être pris en compte pour contribuer à distinguer les liens mentionnés. Nous identifions comme interlocuteurs directs les auteurs et écoles pour qui l'entreprise constitue l'épicentre de leur objet d'étude. Concrètement, nous examinons de manière critique les positions des auteurs post-keynasiens, régulationnistes, marxistes, institutionnalistes ainsi que celles d'autres auteurs dont les travaux relèvent de l'économie de l'entreprise. Cet examen critique de la littérature sur le sujet incorpore de plus une proposition d'interprétation et une mise en contraste empirique appropriée aux cas particuliers de l'économie des États-Unis et de celle de la France qui permettent de mieux fonder nos conclusions.

Mots clés: finaciarisation, relation salariale, actionnariat des salariés, taux de profit et accumulation.

\section{Resumo}

O presente trabalho revisa o debate e a controvérsia atual sobre os laços que relacionam o processo de financiarização econômica com a relação salarial. Nosso objetivo é identificarmos e valorizarmos as fraquezas e carências de tal debate e destacar quais aportações e elementos relevantes podem se considerar para contribuírem à clarificação de tais laços. Identificamos como interlocutores diretos aos autores e escolas que colocam na empresa o epicentro do seu objeto de estudo. Concretamente se revisam de forma crítica as posições dos autores pós-keynesianos, regulacionistas, marxistas, institucionalistas além de outros autores procedentes do campo da economia da empresa. Esta revisão crítica da literatura adiciona, além do mais, uma proposta interpretativa e um contraste empírico próprio para os casos das economias dos Estados Unidos e da França que nos permite fundamentarmos melhor as nossas conclusões.

Palavras chave: financiarização, relação salarial, acionariado salarial, taxa de benefícios e acumulação. 


\section{Introducción y delimitación del debate}

lo largo de las últimas tres décadas, los países de la Organización para la Cooperación y el Desarrollo Económicos (OCDE) han procedido a un rápido pro-

ceso de liberalización de sus mercados financieros. Este hecho ha colaborado - junto con otros fenómenos, entre los que destaca la notable internacionalización del capital financiero a lo largo de este periodo- a que los mercados financieros internacionales hayan adquirido una dimensión y una profundidad espectacular. Ambos factores, a su vez relacionados entre sí, constituyen la base de un nuevo contexto económico internacional financiarizado que influye poderosamente en la dinámica macroeconómica de las distintas naciones, tanto desarrolladas como subdesarrolladas. Buena prueba de ello son las fuertes restricciones que los mercados financieros internacionales imponen a los diferentes Estados desde el punto de vista de la política económica, o el recurrente estallido de crisis financieras y cambiarias a lo largo de los últimos años, tanto en los países desarrollados [crisis del Sistema Monetario Europeo en 1992-1993, naciones escandinavas en 1993, Estados Unidos (EU) en 2001 y en 2008], como en los subdesarrollados (México en 1994, sureste asiático en 1996-1997, Rusia en 1998, Brasil en 1998-1999, Turquía en 2000, Argentina en 2001-2002).

El particular desarrollo que a lo largo de las últimas décadas han experimentado los mercados financieros internacionales - y que ha dado lugar a lo que la literatura especializada denomina "financiarización" - ha llevado a diversos autores a plantearse la incidencia de dicho proceso sobre la dinámica de funcionamiento general de la economía. Numerosos estudios han tratado de analizar y contrastar no sólo la mencionada expansión de la esfera financiera, sino también sus efectos sobre la dinámica macroeconómica, los episodios de inestabilidad y crisis, la evolución del crecimiento económico y la inversión, así como las políticas económicas aplicadas por el Estado, y otros aspectos más.

No obstante, a pesar de que en muchos de estos análisis la relación salarial juega un papel relevante —explícita o implícitamente-, el estudio de sus transformaciones como consecuencia de la expansión de la esfera financiera ha recibido una atención insuficiente. Además ésta es una característica que atraviesa al conjunto de las escuelas que han centrado su atención en el análisis de la financiarización: todas ellas han abierto una agenda de investigación en la que — desde diferentes perspectivas y propuestas metodológicas - se atribuye una importancia central al papel del nuevo contexto financiero internacional (NCFI) en el funcionamiento de la economía, sin embargo los vínculos entre dicho contexto financiero y la dinámica salarial no son abordados con profundidad.

Vol. 39, núm. 155, octubre-diciembre / 2008

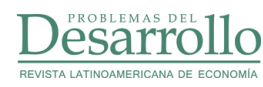


¿Asistimos, tal y como plantean algunos autores (Plihon, 2004), a la transformación de nuestro sistema económico en un "nuevo capitalismo" caracterizado por el poder hegemónico de las finanzas? ¿Es éste el "capitalismo de mañana" del que hablaba Michel Aglietta (Aglietta, 1998) a mediados de los años noventa? ¿Y qué consecuencias tienen estas transformaciones provenientes del ámbito de las finanzas sobre la relación salarial?

Este trabajo se inscribe en ese debate y en la necesidad de contribuir a la clarificación de una cuestión central: ¿cómo se ha modificado la relación salarial como consecuencia del mencionado proceso de financiarización? Su objeto de estudio es la identificación y valoración de las debilidades existentes en dicho debate, resaltando las aportaciones relevantes que contribuyen a la clarificación de los vínculos entre la financiarización económica y la relación salarial. Para ello plantearemos el debate en dos niveles: en primer lugar, revisaremos de manera breve la literatura existente sobre la relación entre el proceso de financiarización y la relación salarial. En segundo, realizaremos una valoración crítica de la literatura revisada, tratando de sugerir y sintetizar una propuesta interpretativa propia para caracterizar los vínculos entre financiarización y relación salarial.

Conviene delimitar los conceptos de los que partimos. Presentamos el difuso término de financiarización económica, que pretende ilustrar — con diferentes matices según los autores (Duménil y Levy, 2004: 110-118; Palazuelos, 1998: 205-207; Ribeiroux, 2003: 26-27; Stockhammer, 2004: 724-28; Martínez González-Tablas, 2007: 269-345; Epstein, 2005: 3-5)— el proceso con el cual desde 1979-1980 emerge la primacía de las finanzas en la dinámica de funcionamiento macroeconómico y en las prioridades de empresas, hogares y administraciones públicas. Dicho concepto recoge por tanto la ascendencia del crecimiento del valor accionarial de las empresas como objetivo prioritario en su gestión, así como la imposición de un nuevo "patrón de acumulación en el cual la realización de beneficios tiene lugar fundamentalmente a través de los canales financieros, en lugar de a través del comercio y la producción de mercancías" (Krippner, 2005: 174). Para efectos de este trabajo, entendemos por relación salarial el conjunto de condiciones económicas e institucionales que regulan y determinan el uso de la fuerza de trabajo, en particular el salario, el tipo de regulación contractual, el nivel de desempleo y la capacidad de negociación colectiva.

Dentro de la controversia que abordamos situaremos como interlocutores los trabajos que, además de atribuir una importancia central al papel del NCFI en el funcionamiento de la economía, sitúan el epicentro de su objeto de estudio en la empresa. Sin pretender un repaso exhaustivo nos concentramos en el papel de las compañías, 
dada la centralidad del vínculo que éstas presentan entre el mercado financiero y la relación salarial. Dicho objeto de estudio forma parte de la agenda de investigación fundamentalmente —aunque no sólo— de enfoques de tradición heterodoxa, como a continuación veremos.

\section{Vínculos teóricos entre la financiarización económica y la relación salarial en las economías desarrolladas: una revisión de la literatura}

En este apartado analizamos las posiciones de los autores poskeynesianos, regulacionistas, marxistas, institucionalistas así como de otros autores procedentes del campo de la Economía de la Empresa. ${ }^{1}$

\section{Escuela poskeynesiana}

Aunque aborda con profundidad las interrelaciones entre el NCFI y los cambios en la dinámica macroeconómica originados por dicho contexto, esta escuela no ha prestado especial atención a la evolución de la relación salarial como tal. Además, el grueso de sus análisis no se desarrolla desde la perspectiva de la empresa y la alteración en sus pautas de funcionamiento, sino desde el análisis de los cambios acontecidos en la interrelación de las distintas variables macroeconómicas. De hecho, el vínculo entre la financiarización de la actividad económica y la relación salarial ha sido analizado por esta escuela siempre de forma subordinada al estudio del crecimiento económico. Así, por ejemplo, Eatwell y Taylor (2000) plantean cómo el nuevo contexto de liberalización y financiarización económica se ha traducido en un fuerte aumento del costo del capital y en una mayor inestabilidad económica y financiera. Estos elementos habrían incidido en una menor inversión empresarial, en una actividad comercial más errática y volátil y, por ende, en un menor crecimiento económico, lo que repercute sobre los niveles de empleo. Esta escuela ha insistido en la mayor capacidad de los sistemas bancarios para promover el crecimiento económico, frente a los que se basan en los mercados financieros (Arestis, Baddeley, y McCombie, 2001), pero destaca el factor negativo sobre la inversión productiva de la volatilidad de estos nichos, así como la privatización — fruto del proceso de financiarización— del riesgo económico (que antes era asumido por el Estado).

1 Remitimos a la bibliografía correspondiente en cada caso para evitar un repaso exhaustivo innecesario.

Vol. 39, núm. 155, octubre-diciembre / 2008

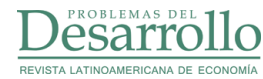


Otros autores poskeynesianos (Kregel, 2004) han recuperado la figura de Minsky para analizar la tendencia autónoma al sobreendeudamiento y a la fragilidad financiera de las economías capitalistas no reguladas, lo que en última instancia desencadena episodios recurrentes de expectativas poco favorables y, por tanto, de reducción de la actividad productiva y de la creación de empleo. ${ }^{2}$

\section{Escuela de la Regulación}

Desde que en 1998 Aglietta publicara Le capitalisme de demain, esta escuela ha situado como punto central de su agenda de investigación el papel de las finanzas en el nuevo "régimen de crecimiento" (Aglietta, 2005 y 2000; Aglietta y Reberioux, 2004; Boyer, 2000; Orlean, 1999). Además, el notable eco que ha tenido esta escuela, sobre todo en Francia, ha llevado a muchos autores a abordar el mismo objeto de estudio con similares categorías teóricas, en lo que se ha llamado el "enfoque del capitalismo patrimonial". ${ }^{3}$ Sus aportaciones en este terreno, y sin ánimo de realizar aquí un resumen, han sido particularmente relevantes: análisis de los principales cambios de los mercados financieros internacionales, del nuevo contexto financiero surgido de dichas transformaciones, de las nuevas formas de propiedad y gestión empresarial, y de la financiarización de las estrategias empresariales. Atribuyen a las finanzas un papel central en el nuevo "régimen de crecimiento", caracterizado por el protagonismo de los mercados bursátiles, el poder de los inversionistas institucionales, la soberanía accionarial que transforma las pautas de funcionamiento empresarial y por la transformación del patrimonio de los hogares. Las finanzas de mercado se constituyen así —en mayor o menor medida según los autores - en el nuevo motor que impulsa las economías desarrolladas (la estadounidense pero también las europeas).

Esta escuela destaca porque — a diferencia de la poskeynesiana — aborda abiertamente las dos dimensiones teóricas que delimitan nuestro objeto de estudio: los vínculos del proceso de financiarización con la relación salarial y una perspectiva centrada en el ámbito de la empresa. Ha dedicado gran atención a la transformación de la relación salarial fruto de la mutación del "régimen de crecimiento": el nuevo modelo liderado por los mercados financieros ha llevado a una progresiva patrimonialización de los ho-

2 Para un análisis más detallado del planteamiento de Minsky, véase Medialdea (2003).

3 Si bien no podemos clasificar a estos economistas (Plihon, Jeffers, Sauviat, Montagne y Ponssard) como integrantes de la Escuela de la Regulación, el "enfoque del capitalismo patrimonial" se articula con base en varios círculos concéntricos, en cuyo seno conviven diferentes autores que comparten interpretaciones en mayor o menor medida con la misma escuela. Para un análisis más detallado de este enfoque véase Álvarez y Medialdea (2008). 
gares asalariados, que se traduce, según la versión más extrema (Aglietta, 1998; Orlean, 1999), en una creciente escisión dentro del trabajo asalariado entre la vertiente salarial y la patrimonial, así como en una creciente socialización de la propiedad del capital y en el desarrollo de "efectos riqueza" que pueden incluso compensar las caídas reales de los salarios. Así, la importancia cuantitativa de los ingresos e incrementos patrimoniales procedentes de fuentes distintas a las del salario se traducirían en "efectos riqueza", que permitirían que el consumo privado pasase a fortalecer sus vínculos con la evolución de la rentabilidad financiera e independizarse de la masa salarial.

\section{Escuela marxista}

Una aportación importante a este debate ha sido aquella que los autores marxistas, en particular los franceses, han llevado a cabo (Chesnais, 2004; Duménil y Lévy 2005 y 2004; Husson 2008 y 2006). Algunos han situado igualmente la financiarización en el centro de su agenda de investigación, si bien con una orientación distinta a la regulacionista. Mientras los regulacionistas mantienen la existencia de una profunda transformación en la relación salarial (creciente esquizofrenia de los trabajadores entre sus ingresos patrimoniales y salariales), los autores marxistas interpretan dichas transformaciones como la respuesta del capital ante la caída de la tasa de ganancia en el marco de la crisis de acumulación iniciada en los años setenta. Así, consideran que los cambios del NCFI sobre la relación salarial se inscriben en un proceso más amplio, caracterizado por el hecho de que el salario ha sido relegado — ante la llegada de la crisis - a variable de ajuste del sistema y, por tanto, a un fuerte retroceso.

En particular, estos autores han situado en la "punción financiera" la forma concreta mediante la cual la financiarización repercute en el proceso de acumulación, en la actividad empresarial y, por ende, en la dinámica salarial. Autores como Chesnais o Duménil y Levy analizan — teórica y empíricamente— la mencionada punción que los mercados financieros efectúan del valor creado por el capital productivo y concluyen que la dimensión alcanzada — vía intereses, dividendos y recompra de acciones - ha determinado la caída del ahorro empresarial y, por tanto, de la tasa de acumulación y de creación de empleo. ${ }^{4}$ Así, la pugna distributiva entre

4 Algunos economistas radicales estadounidenses vinculados al Political Economy Research Institute han adoptado un planteamiento similar, aunque no desde una perspectiva marxista. Es el caso de Crotty (2005), quien analiza la punción financiera en EU, o de Orhangazi (2007), que estudia no sólo la punción financiera, sino también el efecto crowding out que la inversión financiera tiene sobre la inversión productiva en las firmas estadounidenses.

Vol. 39, núm. 155, octubre-diciembre / 2008

$\underline{\text { DeSRarrollo }}$ 
capital productivo y capital financiero repercute directamente sobre el trabajo, que actúa como variable de ajuste. Otros autores marxistas (Husson 2008 y 2006) argumentan que la financiarización no supone una punción de recursos empresariales por parte del capital financiero, sino una huida hacia los mercados financieros de los capitales ociosos que no encuentran vías posibles de rentabilización en el ámbito de la producción: es la sobreacumulación fruto de la hiperconcurrencia, y la consiguiente incapacidad de restaurar la tasa de ganancia, la que desencadena la escasa acumulación empresarial y su negativa repercusión sobre la dinámica salarial.

\section{Escuela institucionalista}

También los autores institucionalistas, particularmente aquellos vinculados al enfoque de "variedades del capitalismo" (Hall y Gingerich, 2001; Hall y Soskice, 2001), se han planteado el objeto de estudio que nos ocupa. Consideran que en las economías desarrolladas contemporáneas existen "esferas institucionales" que se refuerzan y permiten la generación de dinámicas de crecimiento y acumulación — las llamadas "complementariedades"- Destacan las referentes entre el mercado financiero, el mercado laboral y la esfera de la actividad productiva. Estas complementariedades difieren según hablemos de "economías coordinadas de mercado" (Alemania, Francia, Suecia, etcétera) o "economías liberalizadas" (EU, Gran Bretaña, entre otras), pero en todos los casos las complementariedades propias de cada economía dieron lugar después de la Segunda Guerra Mundial a una larga época de expansión, incremento del empleo y crecimiento salarial. No obstante, a partir de las últimas dos décadas comienzan a desencadenarse "desajustes", sobre todo en las economías europeas. Dichos desajustes se producen cuando las "economías coordinadas de mercado" liberalizan sus espacios financieros sin hacer lo mismo con los de trabajo, lo que quiebra las complementariedades y hace desaparecer las dinámicas de acumulación y creación de empleo.

Una crítica especialmente interesante al enfoque de "variedades del capitalismo" proviene del propio ámbito institucionalista. Black, Gospel y Pendleton argumentan en diversos trabajos (Black et al., 2005; Gospel y Pendleton, 2006) que son las instituciones tradicionales del mercado de trabajo (existencia y tipo de negociación colectiva, convenios, grado de afiliación sindical, etcétera) las que explican la evolución de la relación salarial, y no tanto las complementariedades entre los distintos ámbitos institucionales — sobre todo los mercados financieros— - señaladas por el enfoque de "variedades del capitalismo". 


\section{Economía de la Empresa}

Por último, presentan un especial interés aquellos autores que han centrado su análisis en el estudio concreto del funcionamiento empresarial y en los vínculos que el proceso de financiarización tiene con los cambios acontecidos en la gestión empresarial. Tanto autores de extracción marxista (Coutrot, 1998) como próximos a la Escuela de la Regulación, (Montagne y Sauviat 2001a y 2001b; Plihon, 2004; Plihon y Ponssard, 2002) o institucionalistas (Froud et al., 2000; Gospel y Pendleton, 2006; Goyer y Hancké, 2006; Lazonik y O'Sullivan, 2000) han abordado esta cuestión. No obstante, ha sido la propia disciplina de la Economía de la Empresa la que — en ocasiones muy alejada de posiciones heterodoxas - ha establecido con mayor precisión los vínculos concretos entre la financiarización y el funcionamiento empresarial. Estos autores (Batsch, 2002; Beffa, 2000; Bowmann y Singh, 1993; Sentis, 1999 y 1998) han estudiado cómo la financiarización económica ha impulsado a su vez la propia financiarización de las estrategias empresariales, al hacer que se subordinen a los dictados de los mercados bursátiles. Todo un conjunto de estrategias empresariales ha sido desencadenado para garantizar el crecimiento del valor accionarial de la empresa, sobre todo en lo relativo a las estrategias de crecimiento y modificación del perímetro empresarial (recentramiento de la cadena de valor, disminución del activo, subcontratación, deslocalizaciones, fusiones y adquisiciones, entre otros).

\section{Valoración crítica y aportaciones}

más relevantes: una propuesta interpretativa

Una vez enunciadas las principales referencias teóricas que delimitan el debate en el que se enmarca nuestro objeto de estudio, pasamos a realizar una valoración crítica de estas aportaciones, para construir una propuesta analítica propia que colabore en el conocimiento de los vínculos entre financiarización y relación salarial.

Tal y como hemos señalado, los autores poskeynesianos centran su análisis en la dimensión macroeconómica; no analizan de manera directa los vínculos existentes entre el proceso de financiarización económica y la dinámica salarial, sino que — cuando lo hacen - lo plantean como un subproducto derivado del estudio de la inversión y el crecimiento. Esta carencia (dado que este vínculo que no abordan de forma explícita sí juega un papel relevante en su esquema teórico) ha sido parcialmente solventada por aportaciones puntuales. Destacan las de Stockhammer (2006 y 2004), autor que desde una perspectiva de clase sitúa a la 
empresa en el centro de su análisis, identificando el impacto de la financiarización sobre las decisiones de gestión del beneficio y de reinversión, y por tanto sobre la lógica de acumulación, empleo y salario, llegando a conclusiones similares a las de Duménil y Lévy (2004).

Aunque las aportaciones de la Escuela de la Regulación en este terreno han sido relevantes, quizá una de las propuestas que más explícitamente se ha planteado el vínculo entre la financiarización económica y la dinámica salarial sea también una de las que presenta carencias más evidentes. Más allá de las posibles críticas a su interpretación general de la nueva fase financiarizada del capitalismo contemporáneo, ${ }^{5}$ son de particular importancia las debilidades referentes a su interpretación de las transformaciones de la relación salarial. La debilidad de la contrastación empírica en la que Aglietta u Orlean apoyan sus argumentos les permite sostener la existencia de una supuesta "esquizofrenia salarial" en el seno del trabajo. A esta escisión de ingresos en el seno del trabajo le acompañaría además una supuesta escisión de intereses.

La insistencia de estos autores (Aglietta, 2000) — sobre todo para el caso de la economía de EU pero también para el francés- en la importancia cuantitativa de los ingresos e incrementos de patrimonio recibidos por los trabajadores procedentes de fuentes distintas a las del salario — plusvalías bursátiles, incrementos de capital financiero, dividendos, etcétera—, así como en la eficacia de los "efectos riqueza" para dinamizar el consumo con independencia de la evolución de la masa salarial, omiten el hecho de que, según datos de la OCDE, menos de 13\% de los hogares franceses poseía acciones en 2000.

Esta cifra, de acuerdo con el European Savings Institute, asciende hasta 23\%, si se consideran las acciones detentadas mediante fondos de inversión. En cualquier caso, la distribución de la propiedad entre los hogares no se ha modificado sustancialmente: en 2000, sólo $7.4 \%$ de los hogares franceses con ingresos mensuales inferiores a los 1,500 euros tenía acciones, comparado con $11.2 \%$ de aquellos hogares con ingresos mensuales entre 1,500 y 2,300 euros, y con 14.3\% de aquéllos entre 2,300 y 3,050 euros (OCDE, 2002).

Además, un estudio del Observatorio del Accionariado Salarial en Europa determina que los trabajadores asalariados eran dueños sólo de $2.6 \%$ de la capitalización de la Bolsa de París en 2000 (Trebucq, 2001). Incluso en EU —donde el

5 Véanse las críticas de Chesnais (2004) y de Husson (2008). 
número de hogares con acciones en bolsa (directamente o por medio de fondos de inversión) se eleva hasta casi la mitad - no se puede ignorar que $85 \%$ de las acciones del país, y $89 \%$ de los títulos financieros, estaba en manos de $10 \%$ de la población en 2001 (Wolf, 2004: tabla 6). Es más, 44\% de estas acciones y de los fondos de inversión, y 58\% de los títulos financieros, pertenecía al 1\% más rico de la población. Incluso tal y como señalan Maki y Palumbo (2001), para la economía estadounidense los hogares para los que se contrasta un aumento de la propensión marginal a consumir durante los años noventa son apenas $20 \%$ de hogares con los ingresos más elevados.

En otras palabras, "todo el boom del consumo realmente puede ser atribuido a los grupos de hogares más ricos" (2001:22). Dew-Becker y Gordon (2005) analizan cómo en EU la masa salarial ha experimentado una notable caída sobre la renta nacional (pasando de 69\% en 1980 a $62 \%$ en 2005) cuando del cálculo de dicha masa se detraen los salarios de $1 \%$ más rico de asalariados (la renta nacional que va a $1 \%$ de los asalariados mejor pagados ha pasado de $4.4 \%$ a $8 \%$ entre 1980 y 2005 , es decir, una captación de 3.5 puntos del PIB). Las ganancias de productividad han sido captadas por tanto, además de las rentas del capital, por una delgada capa de beneficiarios de muy altos salarios.

Los "efectos riqueza" puede que operen para algunas capas de población, pero no para la mayoría de la masa asalariada. Por tanto, tratar de vincular los "efectos riqueza" con una recomposición general de la relación salarial constituye un salto teórico sin fundamento.

Sin duda se han producido importantes transformaciones en la relación salarial de las economías desarrolladas a lo largo de las últimas tres décadas, pero no en la dirección apuntada por la Escuela de la Regulación. De hecho, el vector director que ha presidido estas transformaciones no es tanto la modificación parcial aunque creciente de la lógica patrimonial de los hogares asalariados, sino — como sugiere la interpretación marxista- la degradación progresiva y sistemática de la relación salarial en la que se inscribe el trabajo tras la Segunda Guerra Mundial, como se ilustra a continuación.

En primer lugar, se ha dado una destrucción relativa y una sustitución de puestos de trabajo, tanto en la economía francesa como en la estadounidense. En el primero de los casos, la tasa de desempleo pasó de 2\%-3\% durante los años setenta a situarse de media por encima de $10 \%$ desde los años ochenta hasta ahora. Una tendencia similar se observa para la economía de Estados Unidos durante los años ochenta. Además, a pesar de que durante los años noventa la tasa de desempleo 
disminuye en este país, continúa la destrucción y sustitución de puestos de trabajo: la tasa de pérdidas de empleos (media anual de trabajos perdidos sobre el total de la fuerza de trabajo) se situó en cerca de $14 \%$ durante la primera mitad de los años noventa (Farber, 1997). Y esta tendencia continúa durante la segunda mitad de los noventa, como señalan Lazonick y O'Sullivan (2000), al producirse una sustitución de trabajos estables y bien pagados por otros temporales y precarios.

En segundo lugar, se ha producido un transvase de riesgo hacia los asalariados. En EU 35\% de los trabajadores despedidos seguía desempleado tras dos años, y aquellos que encontraban un nuevo puesto de trabajo presentaban de media un salario 13\% inferior (Lazonick y O'Sullivan, 2000:20). En Francia, el empleo temporal ha pasado de $3.3 \%$ en 1983 a $12.8 \%$ en 2006, mientras que el empleo a tiempo parcial creció de $9.6 \%$ a $17.3 \%$ durante el mismo periodo (siendo el porcentaje de empleo a tiempo parcial involuntario 30\% del total). La tasa de transición entre empleo temporal y empleo indefinido cayó de 40\% en 1989 a 33\% en 2001 (Enquête Emploi 1989-2002, INSEE), y la tasa de rotación laboral pasó de 22\% a 36\% entre 1983 y 2002 en las empresas francesas de más de 50 empleados.

Además se ha desencadenado durante las últimas décadas una significativa congelación salarial. Francia y EU vuelven a ser buen ejemplo de ello, como se ve en el cuadro 1: los salarios han dejado de crecer en ambas economías al ritmo al que lo hace la productividad (como sucedía en los años cincuenta y sesenta).

\section{Cuadro 1}

Productividad y salarios reales, sector privado, EU, Francia 1950-2005 (tasa de crecimiento anual acumulativa \%)

\begin{tabular}{lrcccccc}
\hline & & $1950-1959$ & $1960-1969$ & $1970-1979$ & $1980-1989$ & $1990-1999$ & $2000-2005$ \\
\hline \multirow{4}{*}{ EU } & Productividad & 2.3 & 2.9 & 1.9 & 1.6 & 2.0 & 3.0 \\
& Salancias & 2.5 & 1.8 & -0.1 & -0.4 & 0.5 & 0.3 \\
& Productividad & - & 6.7 & 3.8 & 3.0 & 1.7 & 1.5 \\
& Salarios & - & 4.2 & 4.7 & 1.3 & 1.0 & 1.4 \\
\hline
\end{tabular}

Fuente: Elaboración propia a partir de Bureau of Labor Statistics (2007) e INSEE (2007).

El hecho de que en estas economías los salarios reales hayan registrado un ritmo de crecimiento notablemente inferior al de la productividad ha supuesto un importante proceso de redistribución de la renta nacional, lo que hace que retroceda el peso de las rentas salariales sobre el PIB, tal como se aprecia en la gráfica 1. 
Gráfica 1

Remuneración de asalariados, EU y Francia, 1978-2005 (\% PIB)

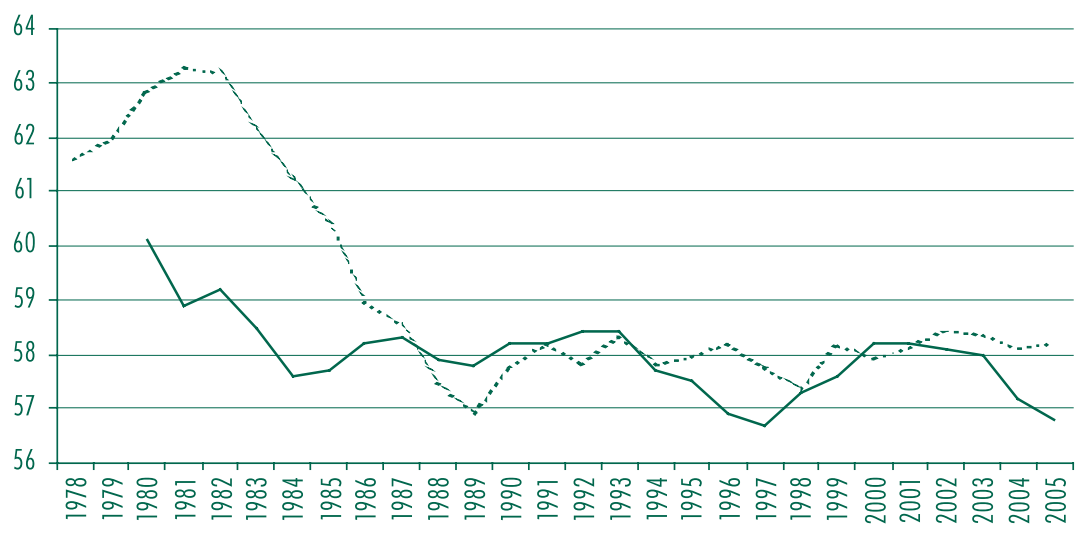

- EU

Fuente: Elaboración propia a partir de BEA, National Economic Accounts (2007); INSEE, Comptes nationaux annuels (2007).

La interpretación marxista constituye un referente que permite identificar mejor el sentido general de estas transformaciones, tanto en el plano general como en el análisis concreto de la relación que éstas mantienen con el proceso de financiarización. Las transformaciones contemporáneas del trabajo se inscriben según esta escuela (Arrizabalo, 1997; Chesnais, 2004; Duménil y Lévy, 2005 y 2004; Husson, 2008) en la órbita de las contrarreformas asociadas al ajuste ante la crisis de rentabilidad que atraviesa el sistema capitalista desde los años setenta. La financiarización de la economía y la "punción financiera" son los instrumentos concretos de los que se ha dotado el capital en dicho proceso de contrarreformas para restaurar la tasa de rentabilidad. Destacan los análisis de Duménil y Lévy en la medida en que aportan una excelente fundamentación empírica de este razonamiento.

Al replicar cálculos análogos a los desarrollados por estos autores (Duménil y Lévy 2005 y 2004) para evaluar su argumentación, llegamos a conclusiones similares. Al final de los años sesenta los países desarrollados comenzaron a experimentar una caída de la tasa de beneficio, que apenas unos años más tarde se traduciría en un cortocircuito en la acumulación y una crisis económica generalizada. La sobreinversión empresarial progresivamente minó la rentabilidad marginal de nuevas inversiones, lo que al final precipitó el mencionado cortocircuito en la acumulación y el inicio de una larga fase de crecimiento débil, elevado desempleo y fuertes déficit públicos. 
En este contexto, el capital ha protagonizado una poderosa ofensiva a lo largo de las últimas tres décadas para propiciar la recuperación de la rentabilidad empresarial (Onaran, 2005; Duménil y Lévy, 2004). Esta ofensiva, que al igual que la crisis ha tenido y tiene en la actualidad una dimensión mundial, se ha dotado de diversos instrumentos con el objetivo fundamental de recuperar los beneficios (Arrizabalo, 1997 y Onaran, 2005): la apertura externa de las distintas economías nacionales al capital transnacional, la privatización progresiva de los sectores productivos y de las coberturas sociales públicas, y la liberalización y desregulación de los mercados. La tasa general de beneficios se ha recuperado en EU y Francia gracias a esta ofensiva: según nuestros cálculos, en 1982 esta tasa era de 6.4\% en EU y de 10.4\% en Francia; en 2006 su valor era de $13.8 \%$ para EU y de $14.5 \%$ para Francia.

Estas medidas neoliberales —encaminadas a ensanchar y profundizar los espacios de valorización del capital— han propiciado la transformación de la relación salarial a la que antes se aludía. Destacan la liberalización y desreglamentación del mercado de trabajo, y la eliminación del "corsé" que el periodo keynesiano había impuesto al desarrollo especulativo y cuasi autónomo de las finanzas. Ambas medidas, junto con las antes señaladas, han permitido un desarrollo extraordinario de la esfera financiera. El enfoque del capitalismo patrimonial destaca acertadamente el papel protagonista del ascenso del capital financiero en la recomposición de la economía mundial en estos 30 años. No obstante, no termina de identificar de manera correcta la dirección del movimiento desplegado por las finanzas. Como ha señalado Husson (2006), el objetivo último de este movimiento ha sido la utilización de esta esfera como palanca de recomposición social entre clases para conseguir el objetivo de la recuperación de la rentabilidad.

Nuestros propios cálculos también confirman la tesis marxista de la "punción" de recursos ejercida por los mercados financieros en las economías tomadas aquí como ejemplos. La esfera de las finanzas se ha servido fundamentalmente de tres instrumentos para detraer recursos económicos de la esfera productiva (Crotty, 2005; Duménil y Lévy, 2004): altos tipos de interés reales, aumento del porcentaje de beneficio empresarial que es destinado a dividendos y mecanismos de recompra de acciones por parte de las propias empresas. La superposición de los tres mecanismos ha supuesto una reducción en la tasa de beneficio retenido por las empresas. En la medida en que ésta es una variable clave para explicar la inversión empresarial, ello se ha traducido a su vez en menores ritmos de acumulación durante las últimas tres décadas, menores ritmos de creación de empleo y mayores tasas de desempleo. 
Los casos estadounidense y francés ilustran bien esta tendencia de la tasa de beneficio, como puede verse en las gráficas 2 y 3 . En ambas podemos distinguir cómo las políticas neoliberales han restaurado la tasa de beneficio de las sociedades no financieras (antes del pago de intereses y dividendos). Sin embargo, la tasa de acumulación no ha experimentado una recuperación similar en ninguno de los dos países, y una de las razones que explica esta tendencia es el hundimiento de la tasa de beneficio retenido por las sociedades. ${ }^{6}$

Gráfica 2

Tasa de beneficio, tasa de beneficio retenido y tasa de acumulación, sociedades no financieras, EU, 1948-2006 (\%)

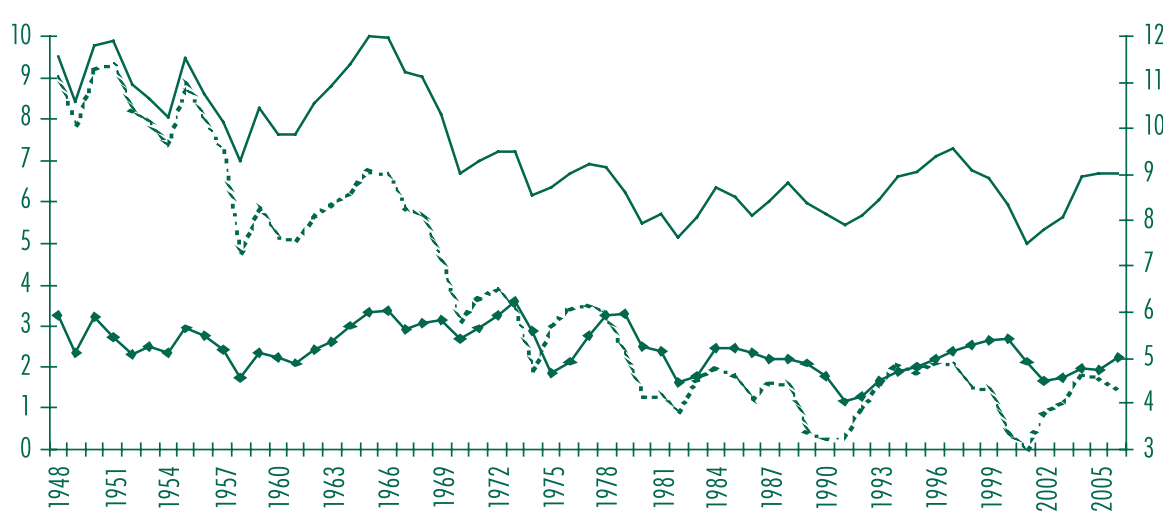

Tasa de beneficio $\quad$........ Tasa de beneficio retenido $\quad \longrightarrow$ Tasa de acumulación

Fuente: Elaboración propia a partir de BEA, National Economic Accounts (2007).

Dicha tendencia contrasta la tesis marxista de la punción financiera e ilustra cómo las finanzas han actuado como vehículo de redistribución de ingresos entre distintas clases sociales, permitiendo una recuperación de la rentabilidad general del capital

6 La tasa de beneficios retenidos de las sociedades no financieras se define aquí teniendo en cuenta sólo los dividendos e intereses pagados, y no los intereses y dividendos recibidos por éstas. Tratamos así de separar dos fenómenos simultáneos y relacionados con la financiarización de las estrategias empresariales. Por un lado, el proceso que tratamos de aislar en nuestra definición de beneficio retenido: flujos de intereses y dividendos asociados con la actividad de explotación habitual de la empresa. Una variable "proxy" de estos flujos son las salidas que se producen (dividendos e intereses pagados por las sociedades). Por otro, excluimos en nuestros cálculos los flujos de entrada (dividendos e intereses recibidos por las sociedades), la mayoría de los cuales está relacionado con la reorientación de los recursos de las sociedades no financieras hacia destinos financieros en lugar de productivos.

Vol. 39, núm. 155, octubre-diciembre / 2008

$\frac{\text { DeSRarrollo }}{\text { PRo }}$ 
Gráfica 3

Tasa de beneficio, tasa de beneficio retenido y tasa de acumulación, sociedades no financieras, Francia, 1978-2006 (\%)

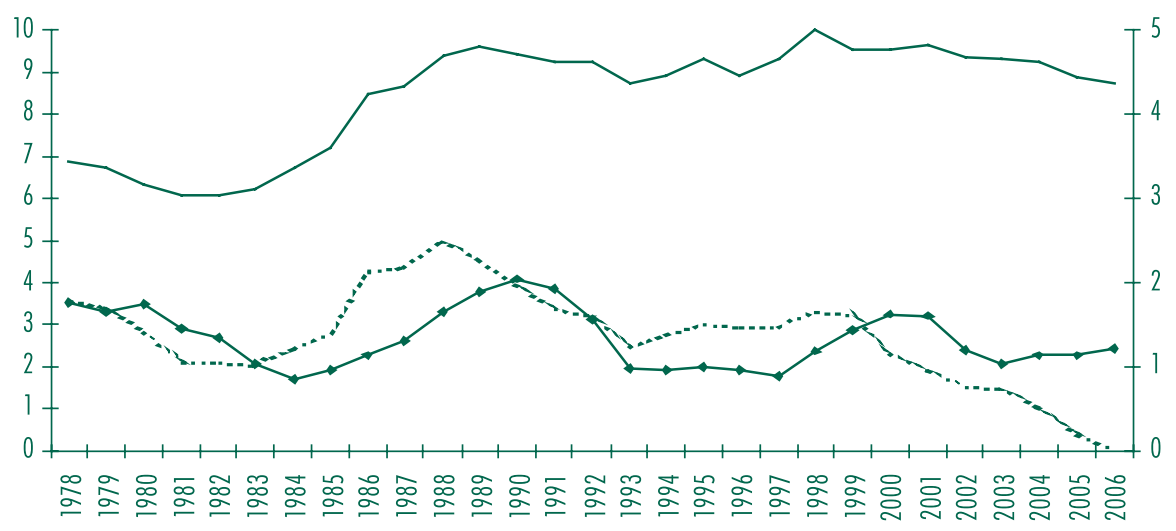

- Tasa de beneficio $\quad$....... Tasa de beneficio retenido $\longrightarrow$ Tasa de acumulación

La tasa de beneficio y la tasa de beneficio retenido se representan en la escala de la izquierda. La tasa de acumulación se representa en la escala de la derecha. Ambas escalas han sido normalizadas en una escala de 0 a 10.

La tasa de beneficio se define en estas dos gráficas como los beneficios empresariales divididos por el stock neto de capital fijo de las sociedades. La tasa de beneficio retenido se define como beneficios empresariales menos los dividendos y los intereses pagados, dividido por el stock neto de capital fijo de las sociedades. La tasa de acumulación se define aquí como el cociente de la inversión neta de capital fijo por el stock neto de capital fijo. Las tres tasas tienen en cuenta sólo datos de las sociedades no financieras.

Fuente: Elaboración propia a partir de INSEE, Comptes Nationaux Annuels (2007).

a expensas de la inversión empresarial y los niveles de empleo. En este sentido, la crisis que durante 2008 ha eclosionado en el ámbito mundial no es más que una nueva expresión - especialmente destructiva - del descontrolado poder de las finanzas y de la capacidad del capital financiero para hacer valer sus intereses.

Las dificultades de valorización de la actividad productiva y la enorme abundancia de liquidez resultante, junto con la desreglamentación y liberalización financiera internacional y el pinchazo bursátil de 2001, propiciaron entre 2001 y 2007 la activación de una de las inercias más consustanciales a la lógica capitalista: la formación de una burbuja especulativa, centrada en este caso en torno a los precios de los inmuebles, el mercado hipotecario y el mercado de derivados (donde se han negociado millones de operaciones vinculadas a las hipotecas subprime).

La formación de esta ingente masa de capital ficticio se derrumbó una vez que los agentes económicos percibieron la desconexión entre los precios de las viviendas en la 
economía estadounidense y el valor real de dichos activos, propiciando el incremento de la morosidad de las hipotecas más arriesgadas, la desvalorización de los paquetes financieros que habían titularizado dichas hipotecas y, con ello, los problemas en los balances de las instituciones financieras. A partir de ahí, esta crisis - fruto de la liberalización financiera y de la tendencia intrínseca del capitalismo a desarrollar burbujas especulativas construidas sobre la formación de una ingente masa de capital ficticio sin relación con activos reales- no hace sino reforzar los efectos que antes apuntábamos: las finanzas han actuado como verdadera palanca de redistribución de ingresos y de recomposición social. El estallido de la crisis hipotecaria y financiera ha desencadenado a su vez una fuerte restricción del crédito, fruto de la incertidumbre de los bancos al desconocerse qué agentes están infectados por activos "tóxicos" (hipotecas subprime). Y es precisamente esta fuerte restricción del crédito —originada en definitiva por la capacidad del capital financiero de poner la economía mundial al servicio de sus necesidades de valorización — la que ha desencadenado el traspaso de la crisis financiera a la actividad productiva, restringiendo enormemente la inversión empresarial y el consumo privado.

Además, el rescate económico diseñado por la administración estadounidense — centrado en la compra de los activos "tóxicos" por parte del Estado- no es sino una socialización encubierta de las pérdidas de aquellas instituciones financieras que propiciaron y alimentaron el proceso de especulación financiera. Se refuerza también así la transferencia de rentas desde los asalariados hacia el capital financiero.

Como vemos, esta crisis reforzará de manera notable — dada su enorme magnitud- el proceso de ralentización desplegado por el fenómeno de punción financiera antes analizado. Además, seguramente modificará las propias relaciones en la actualidad existentes entre el capital financiero internacional y las empresas no financieras.

No obstante, y dejando de lado el análisis particular de la crisis financiera de las hipotecas subprime, los esfuerzos que desde el marxismo se han hecho para relacionar en las economías desarrolladas el proceso de financiarización actual con la relación salarial —al igual que en el caso de algunas aportaciones poskeynesianas como las de Stockhammer — hacen bascular todo el análisis sobre las políticas de inversión empresarial y la repercusión de éstas sobre la acumulación, el empleo y la dinámica salarial. Se obvia así el impacto global que la financiarizacion tiene sobre la empresa más allá de las políticas de inversión que ésta siga. De hecho, la empresa —entendida como "campo de batalla" en la apropiación por el valor producido y en donde la capacidad de negociación es una variable central (Stockhammer, 2006: 212)—se ve afectada por la financiarización económica también en el terreno de las políticas 
de gestión de la fuerza de trabajo, de crecimiento empresarial, de redefinición del perímetro empresarial, etcétera, lo cual debe tenerse en cuenta.

El enfoque institucionalista de "variedades del capitalismo" presenta el interés de fundamentar su línea argumental sobre el análisis comparativo de diversos estudios de caso - "economías coordinadas de mercado" versus "economías liberalizadas"No obstante, dicha perspectiva es estática, por lo que impide analizar con propiedad la dinámica evolutiva que se haya detrás de las interrelaciones entre la "esfera de las finanzas" y la "esfera del mercado de trabajo". Este tipo de análisis permite únicamente comprobar un determinado grado de correlación en las interrelaciones que se dan entre ambas esferas. De hecho, esta debilidad lleva a este enfoque a no percibir que los "desajustes" entre las distintas esferas, propios de las "economías coordinadas de mercado" (Alemania, Francia, Suecia, etcétera), no se pueden presentar como una variable exógena en el análisis de la financiarización. Responden, como hemos visto, a una profunda corriente de transformaciones estructurales que atraviesan al sistema capitalista en la actualidad (liberalización de los mercados financieros, privatización de los sistemas públicos de pensiones, etcétera), determinando el propio curso de las relaciones entre las nuevas finanzas de mercado y la evolución de la esfera salarial.

Como ya dijimos, han sido los autores del ámbito de la Economía de la Empresa quienes en ocasiones han establecido con mayor precisión los vínculos concretos entre el proceso de financiarización económica y la modificación del funcionamiento empresarial. Han analizado cómo la entrada de los inversionistas institucionales y demás inversionistas financieros en el capital social de las principales empresas han provocado que adopten medidas acordes con sus exigencias, como la reestructuración del perímetro empresarial, dándose un doble proceso: por un lado mientras las organizaciones se desprenden de aquellas partes de su ciclo productivo que menos valor añaden (mediante estrategias de subcontratación y deslocalización) y concentran su actividad en el corazón productivo (estrategias de recentramiento), por otro desarrollan estrategias de crecimiento externo para convertirse en líderes de un determinado segmento de mercado (fusiones y adquisiciones).

Esta línea de investigación resulta sobre todo interesante para adentrarse en el estudio de los vínculos entre la financiarización y la relación salarial. Un ejemplo ilustrativo es el caso francés. Según las investigaciones desarrolladas por diversos autores a partir de la base de datos Sisife-Lerep de la Université de Toulouse (Morin, 2000; Morin y Rigamonti, 2002), la entrada de inversionistas institucionales en el capital de las empresas francesas les ha llevado a constituirse en el principal núcleo de accionistas. Como podemos ver en el cuadro 2, los inversionistas institucionales 
presentan participaciones muy relevantes en el capital de las empresas del CAC 40, oscilando en torno a $25 \%$ de su capital social, con un mayor peso de los inversionistas extranjeros que nacionales. El cuadro 2 muestra además cómo la evolución de la propiedad accionarial de las empresas del CAC-40 entre 1997 y 2001 no ha hecho sino reforzar esta tendencia: la propiedad de los inversionistas institucionales extranjeros ha crecido $66.9 \%$ durante estos años, mientras que la propiedad familiar caía $-4.4 \%$ y la de la banca francesa lo hacía en $-74.4 \%$.

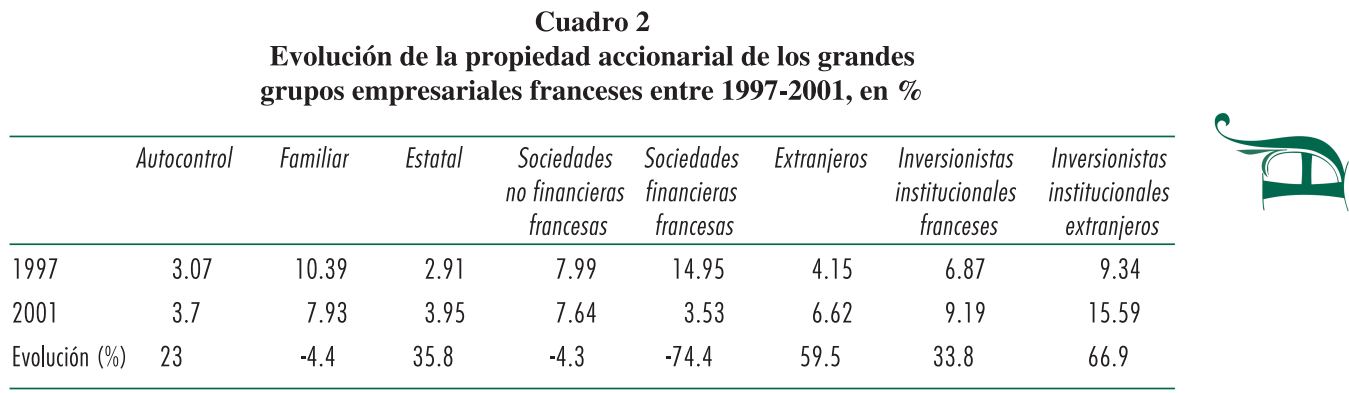

Fuente: Base de datos sobre propiedad accionarial de Sisife-Lerep, Université de Toulouse, LEREPS; tomado de Morin y Rigamonti (2002)

Tal y como plantean Goyer y Hancké (2006), la llegada de los inversionistas institucionales al accionariado de las empresas francesas ha modificado profundamente las estrategias de éstas, en la línea en la que antes apuntábamos: progresiva eliminación de los conglomerados y creciente recentramiento estratégico sobre aquellas actividades de base de la empresa en las que ésta tiene ventajas comparativas con respecto a sus competidoras. ${ }^{7}$

Como vemos en el cuadro 3, el tejido productivo francés se ha visto atravesado por una profunda reestructuración entre 1994 y 2002 con respecto a las estrategias empresariales de los diferentes grupos. Muchas grandes firmas francesas han reducido de manera sustancial su grado de diversificación en beneficio de una mayor focalización de su negocio. En 1986, 83\% de las principales sociedades francesas del CAC 40 tenían una estrategia de negocio diversificada, mientras que en 1998 dicho porcentaje ya había descendido a 52\%, y a 45\% en 2002.

7 Para una explicación más detenida de las causas por las cuales los mercados financieros presionan para imponer estas estrategias, véase Goyer y Hancké (2006) y Morin y Rigamonti (2002). 
Cuadro 3

Evolución de las estrategias corporativas de las principales sociedades francesas entre 1986-2002, en \%

\begin{tabular}{lccccc}
\hline Tipo de estrategia empresarial & $\begin{array}{c}1986 \\
\text { (30 empresas) }\end{array}$ & $\begin{array}{c}1990 \\
\text { (30 empresas) }\end{array}$ & $\begin{array}{c}1994 \\
\text { (30 empresas) }\end{array}$ & $\begin{array}{c}1998 \\
\text { (30 empresas) }\end{array}$ & $\begin{array}{c}2002 \\
\text { (30 empresas) }\end{array}$ \\
\hline Negocio diversificado & 83.3 & 78.1 & 75.0 & 51.6 & 44.8 \\
Línea de negocio dominante & 3.3 & 9.4 & 9.4 & 29.0 & 31.0 \\
Única línea de negocio & 13.3 & 12.5 & 15.6 & 19.4 & 24.1 \\
\hline
\end{tabular}

Fuente: Goyer y Hancké (2006:188).

No obstante, estos autores del ámbito de la Economía de la Empresa — por la propia condición y naturaleza de sus objetos de estudio — no han dado el paso para analizar con detalle el impacto del cambio en el funcionamiento empresarial inducido por el NCFI sobre la dinámica salarial, y sólo se han limitado al análisis de dichas estrategias empresariales. Así, aquellos casos en los que se abordan los vínculos del proceso de financiarización con las políticas empresariales de gestión de la fuerza de trabajo apenas se limitan a estudiar los problemas de agencia entre accionistas y directivos, así como las prácticas seguidas para atenuar estas supuestas divergencias (stock options, participación en beneficios, etcétera).

Sin embargo, esta modificación del perímetro empresarial promovida — vía corporate governance - por los inversionistas financieros va a tener importantes consecuencias para la fuerza de trabajo: en primer lugar, se traduce en un deterioro de los niveles de empleo (Lazonick y O’Sullivan, 2000); además, la modificación del perímetro empresarial supone la supresión de los mercados internos de trabajo de las grandes empresas y, por tanto, de las posibilidades de movilidad, progresión y protección que éstos suponían; por último, también supone una ruptura de los espacios laborales tradicionales y la determinación de una menor capacidad de negociación frente al capital.

Han tenido que ser otros autores, provenientes de otras escuelas, quienes utilicen los desarrollos de la Economía de la Empresa para establecer el vínculo entre el proceso de financiarización y la modificación de las relaciones laborales. Es el caso del interesante trabajo empírico sobre la relación entre los mercados financieros y la participación de los asalariados en las decisiones empresariales de Reberioux (2003), que muestra cómo aquellas empresas francesas que cotizan en bolsa, y que por tanto están sometidas a la influencia de los mercados financieros, proporcionan a sus asalariados menos información de la situación económica de la firma y menos capacidad para incidir sobre la estrategia, el cambio organizacional o el empleo, que las que no cotizan en los mercados de capitales. Así este autor contrasta una menor capacidad 
de toma de decisiones para los asalariados y sindicatos franceses de aquellas compañías que desarrollan cambios organizacionales (recentramiento de la cadena de valor, externalizaciones, fusiones y adquisiciones, etcétera).

Además Rebeiroux apunta cómo la nueva presencia de los inversionistas institucionales extranjeros en el seno de las empresas francesas se hace en detrimento de la capacidad de las organizaciones sindicales. Por todo ello, considera que la financiarización de las estrategias empresariales supone una recomposición en la correlación de fuerzas en beneficio del capital financiero y conlleva un efecto negativo sobre la calidad de la información proporcionada a los asalariados y sobre la integración de éstos en la toma de decisiones de la empresa, lo que hace frágil su posición.

Por tanto, son dos las líneas de investigación que identificamos en este apartado como prioritarias de cara a construir una propuesta analítica propia que aborde la caracterización de los vínculos entre financiarización y relación salarial. En primer lugar, el estudio de la punción financiera, que determina la modificación de las estrategias inversoras de las empresas y, por tanto, el nivel de acumulación y de empleo. En segundo, la línea de investigación vinculada con las estrategias de reestructuración del perímetro empresarial (subcontratación, deslocalización, recentramiento, etcétera) y su relación con las presiones de los mercados financieros.

Ambas líneas de trabajo abren perspectivas interesantes - y convergentes- para comprender mejor cómo la financiarización económica repercute sobre los aspectos salariales e institucionales de la relación salarial.

\section{Balance y conclusiones}

Con este trabajo hemos intentado ubicar y contextualizar el debate sobre la naturaleza de los vínculos existentes entre el proceso de financiarización económica y las mutaciones experimentadas por la relación salarial en estas últimas décadas, con la idea de situar las principales controversias y carencias de dicho debate, y así contribuir a su clarificación. Comprobamos que a la fecha el debate sigue abierto e inconcluso, que precisa aún de mucha discusión (sobre todo en las circunstancias que parecen abrirse hoy día con la crisis mundial desencadenada por el pinchazo de la burbuja inmobiliaria y financiera).

En concreto, la caracterización que algunas de las escuelas aquí analizadas hacen de estos vínculos puede llegar a ser incluso contradictoria entre sí, como es el caso de la escuela regulacionista y la escuela marxista: mientras que para la primera de ellas —en su versión más extrema representada por Aglietta— el proceso de financiarización económica puede llegar a suponer una mejora de las rentas percibidas por los 
asalariados (dada su patrimonialización financiera), para la segunda de las escuelas el proceso de financiarización representa la comprobación de una regresión salarial.

El excesivo énfasis que hace la Escuela de la Regulación en la creciente entrada de los asalariados en los mercados financieros y bursátiles (acuñándose incluso el concepto de accionariado salarial) impide encuadrar este proceso en su verdadera dimensión teórica: el creciente recurso de los asalariados a los fondos de pensiones, los fondos de inversión o directamente a los títulos bursátiles no supone más que un pivotar desde formas tradicionales de ahorro salarial (patrimonio inmobiliario, depósitos bancarios a la vista, de ahorro, cuentas vivienda, etcétera) hacia otras formas de ahorro salarial más rentables dado el funcionamiento de la economía mundial actual. A pesar de que esta reorganización del ahorro salarial de los trabajadores sea sumamente relevante para entender el nuevo esquema de funcionamiento macroeconómico de los países de la OCDE, no introduce, desde el punto de vista teórico, ninguna novedad relevante en cuanto a la conceptualización del trabajo asalariado. El trabajo asalariado sigue dependiendo en estas economías de la venta diaria de su fuerza de trabajo para subsistir, dependencia que no puede ser superada a partir de la gestión ni de la financiarización de su ahorro, cualquiera que sea la forma que dicha gestión tome. Además, en su intento por aumentar su ahorro salarial, el trabajo asalariado sigue viendo cómo éste es administrado en beneficio propio por los distintos gestores posibles (bancos, fondos de pensiones, fondos de inversión, etcétera), al igual que pasaba en periodos anteriores.

Por otro lado, la escuela marxista, a pesar de que critica con dureza el planteamiento regulacionista (Husson, 2006), no ha acometido la tarea de contrastar empíricamente la magnitud de los supuestos "efectos riqueza" derivados de la financiarización del ahorro salarial, ni tampoco ha analizado la importancia cuantitativa de los ingresos e incrementos de patrimonio recibidos por los trabajadores procedentes de fuentes distintas a las del salario. Sin embargo, esta escuela incorpora una interesante perspectiva de cara al análisis del objeto de estudio aquí presentado, al abordar el proceso de detracción de recursos que experimenta la esfera productiva en beneficio de la financiera.

El resto de escuelas revisadas, a pesar de que no analizan con tanto detalle las relaciones entre el proceso de financiarización y la relación salarial, abren también líneas de trabajo de singular importancia. Destaca particularmente el análisis proveniente de la Economía de la Empresa que aquí hemos analizado: los mercados financieros demandan una serie de estrategias a los grupos cotizados en bolsa que modifican de manera sustancial su perímetro empresarial. Aunque el análisis termina en este punto para estos autores, abre una línea de trabajo muy relevante para conceptualizar las posibles consecuencias que esta redefinición del perímetro empresarial pueda tener 
sobre la relación salarial. Los trabajos de Batsch (2002), o los de Sentis (1999 y 1998), constituyen por tanto una buena base para comenzar a articular una propuesta analítica que caracterice los vínculos entre el proceso de financiarización y los cambios acontecidos en la relación salarial.

\section{Bibliografía}

Aglietta, Michel, Macroéconomie financière, vol. I, París, La Découverte, 2005.

, "Shareholder value and corporate governance, some tricky questions", en Economy and Society, vol. 29, núm. 1, febrero de 2000.

- Le capitalisme de demain, París, Notes de la fondation Saint- Simon, 1998.

y Antoine Reberioux, Dérives du capitalisme financier, París, Albin Michel, 2004.

Álvarez, Ignacio y Bibiana Medialdea, "Financial Globalization and Labor, Employee Shareholding or Labor Regression?", Working Paper Series, núm. 172, Massachusetts, Political Economy Research Institute, junio de 2008.

Arestis, Philip, Michelle Baddeley y John Mccombie, What Global Economic Crisis?, Londres, Palgrave Macmillan, 2001.

Arrizabalo, Xabier, Crisis y ajuste en la economía mundial, implicaciones y significado de las políticas del FMI y el BM, Madrid, Ed. Síntesis, 1997.

Batsch, Lauren, Le capitalisme financier, París, La Découverte, 2002.

Beffa, Jean-Louis, "Ce que le marché demande à l'entreprise", Sociétal, núm. 28, París, 2000.

Black, Boyd, Howard Gospel y Andrew Pendleton, 'Finance, Corporate Governance, and Labour, Evidence from OECD countries', mimeo, King's College Londres, 2005.

Bowmann, Edward y Harbir Singh, "Corporate restructuring, reconfiguring the firm", Strategic Management Journal, Special Issue, Corporate Restructuring, vol. 14, verano, West Sussex, 1993.

Boyer, Robert, "Is a finance-led growth regime a viable alternative to Fordism? A preliminary analysis", en Economy and Society, vol. 29, núm. 1, febrero de 2000.

Vol. 39, núm. 155, octubre-diciembre / 2008
Chesnais, François, La finance mondialisée. $R a$ cines sociales et politiques, configuration, conséquences, París, La Découverte, 2004.

Coutrot, Thomas, L'entreprise néo-libérale, nouvelle utopie capitaliste?, París, La Découverte, 1998.

Crotty, James, "The Neoliberal Paradox, The Impact of Destructive Product Market Competition and 'Modern' Financial Markets on Nonfinancial Corporation Performance in the Neoliberal Era", en Gerald A. Epstein, Financialization and the World Economy, 2005.

Dew-Becker, Ian y Gordon, Robert J., "Where Did the Productivity Go? Inflation Dynamics and the Distribution of Income", Brookings Papers on Economic Activity, 36 (2), 2005.

Duménil, Gérard y Dominique Lévy, "Costs and Benefits of Neo-Liberalism, A Class Analysis", en Gerald Epstein, Londres, Edward Elgar Publishing, 2005.

, Capital Resurgent. Roots of the Neoliberal Revolution, Massachusetts, Harvard University Press, 2004.

Eatwell, John y Lance Taylor, Global Finance at Risk, Cambridge, Polity Press, 2000.

Epstein, Gerald, Financialization and the World Economy, Londres, Edward Elgar Publishing, 2005.

Farber, Henry S., "The changing face of job loss in the United States 1981-1995", Brookings Papers on Economic Activity: Microeconómics 1997, Washington, DC, 1997.

Froud, Julie, Colin Haslam, Sumar Johal, y Karel Williams, "Restructuring for shareholder value and its implications for labor", Cambridge Journal of Economics, vol. 24, núm. 6, Cambridge, noviembre, 2000.

Gospel, Howard y Andrew Pendleton, Corporate Governance and Labour Management, An International Comparison, Oxford, Oxford University, 2006. 
Goyer, Michel y Bob Hancké, "Labour in French Corporate Governance, The Missing Link", en Gospel y Pendleton, Corporate Governance and Labour Management, An International Comparison, Oxford, Oxford University, 2006.

Hall, Peter y Daniel Gingerich, "Varieties of Capitalism and Institutional Complementarities in the Macroeconomy, An Empircal Analysis", Cambridge, Mimeo, 2001.

y David Soskice, Varieties of Capitalism, Oxford, Oxford University Press, 2001.

Husson, Michel, Un pur capitalisme, París, Editions Page Deux, 2008.

-, "Finance, hyper-concurrence et reproduction du capital", en Brunhoff, Suzanne de et al., La finance capitaliste, Séminaire d'Études Marxistes-Actuel Marx, París, PUF, 2006.

Kregel, "Fragilidad financiera e inestabilidad económica", en Correa, Eugenia y Alicia Girón, Economía Financiera Contemporánea, tomo III, México, Miguel Ángel Porrúa, 2004.

Krippner, Greta R., "The financialization of the American economy", Socio-Economic Review, núm. 3, Oxford, mayo de 2005.

Lazonick, William, y Mary O'Sullivan, "Maximizing shareholder value, a new ideology for corporate governance", Economy and Society, vol. 29, núm. 1, febrero de 2000.

Maki, Dean M., y Michael G. Palumbo, "Disentangling the wealth effect, a cohort analysis of household saving in the 1990s", Finance and Economics Discussion Series, núm. 21, Washington, Board of Governors of the Federal Reserve System, 2001.

Martínez González-Tablas, Ángel, Economía Política Mundial. I Las fuerzas estructurantes, Madrid, Ariel Económica, 2007.

Medialdea, Bibiana, Inestabilidad financiera en las economías emergentes latinoamericanas en los años noventa, el caso de Brasil, Trabajo de Investigación, Facultad de Ciencias Económicas y Empresariales, Universidad Complutense de Madrid, 2003.

Montagne, Sabine y Catherine Sauviat, "L'Impact des marches financiers sur la gestion des ressources humaines, une enquête exploratoire auprès de grandes entreprises françaises", Document d'Etude, núm. 42 , marzo, París, DARES, 2001a.

-, "L'influence des marchés financiers sur les politiques sociales des entreprises, le cas français", Travail et Emploi, núm. 87, París, julio de 2001b.

Morin, François, "A transformation in the French model of shareholding and management", Economy and Society, vol. 29, núm. 1 , febrero de 2000.

y Eric Rigamonti, "Évolution et structure de l'actionnariat en France", Revue Française de Gestion, vol. 28/141, París, 2002.

Onaran, Özlem, "Life After Crisis For Labor And Capital in the Era of Neoliberal Globalization", Working Papers Series, núm. 43, Vienna University of Economics and Business Administration, 2005.

Orhangazi, Özgür, Financialization and the US economy, Northampton, MA, Edward Elgar, 2007a.

Orléan, André, Le pouvoir de la finance, París, Editions Odile Jacob, 1999.

Plihon, Dominique, Le nouveau capitalisme, París, La Découverte, 2004. y Jean-Pierre Ponssard, Montée en puissance des fonds d'investissement. Quels enjeux pour les entreprises?, París, La Documentation française, 2002.

Reberioux, Antoine, "Les marchés financiers et la participation des salariés aux décisions", Travail et Emploi, núm. 93, París, enero de 2003.

Sentis, Patrick, "Pourquoi les décisions de désinvestissement créent-elles de la valeur?", Revue Française de Gestion, núm. 122, París, 1999.

, "Performances à long terme et caractéristiques financières des entreprises qui réduisent leurs effectifs", Finance Contrôle Stratégie, núm. 4, París, 1998.

Stockhammer, Engelbert, "Shareholder value orientation and the investment-profit puzzle", Journal of Post Keynesian Economics, vol. 28, núm. 2, invierno 2005-2006, Nueva York, M E Sharpe, 2006.

, "Financialization and the Slowdown of Accumulation", Cambridge Journal of Economics, núm. 28 (5), Cambridge, 2004.

Trebucq, Stéphane, Réalité de l'actionnariat salarié en 2000 dans les sociétés françaises cotées, París, Observatoire de l'Actionnariat Salarié en Europe, 2001.

Wolff, Edward N., "Recent Trends in Living Standard in the United States", en Wolff, Edward N., What has happened to the quality of life in the advanced industrialized nations?, Massachusetts, Edward Elgar Publishing, 2004. 